of ROS and NO favours the production of peroxynitrite that is capable of nitrosylation of key cellular proteins such as the Ryanodine receptor that has a crucial role in cardiac excitationcontraction coupling. This study provides novel insights into the mechanisms of cardiac damage in diabetes that occur independent of vascular disease through AGEs.

\section{OPTIMISATION OF MEDICAL THERAPY AFTER CARDIAC RESYNCHRONISATION: A NURSING OPPORTUNITY NOT TO BE MISSED}

doi:10.1136/heartjnl-2011-300198.75

${ }^{1} \mathrm{~S}$ J Russell, ${ }^{2} \mathrm{~J}$ Bell, ${ }^{3} \mathrm{~L}$ Edmunds, ${ }^{4} \mathrm{~J}$ Davies, ${ }^{3} \mathrm{H}$ Rose, ${ }^{3} \mathrm{Z} \mathrm{R}$ Yousef. ${ }^{1}$ Wales Heart Research Institute, Cardiff, UK; ${ }^{2}$ Cardiff University, Cardiff, UK; ${ }^{3}$ University Hospital of Wales, Cardiff, UK; ${ }^{4}$ University Hospital Llandough, Cardiff, UK

Introduction Cardiac resynchronisation therapy (CRT) is indicated in patients with left ventricular dysfunction ( $E F \leq 35 \%)$, electromechanical dyssynchrony, and limiting heart failure (HF) symptoms despite optimal medical therapy. In many cases target doses of HF medications prior to CRT are not achieved due to bradycardia and/or limiting hypotension. CRT however provides bradycardia backup and improved haemodynamics, thus providing an opportunity to further optimise HF medical therapies known to confer substantial morbidity and mortality benefits. We conducted the present study to evaluate the potential to further optimise medical treatments in patients receiving CRT within the framework of nurse-led pre and post CRT clinics.

Methods Our unit operates an integrated CRT service with preassessment, implantation, and follow-up components. Pre-assessment and follow-up incorporate dedicated HF nurse clinics to support protocol-driven optimisation of medical therapies. We therefore conducted a retrospective analysis of our CRT database over a 9-month period to quantify the frequency of use, and dose of HF medications ( $\beta$ blockers; $\beta B$, angiotensin converting enzyme inhibitors: ACE-I or angiotensin receptor blockers: ARB, aldosterone antagonists, digoxin, and loop diuretics) before and 6 months after CRT. Total daily dose equivalences within each class of medication (bisoprolol for $\beta B$, lisinopril for ACE-I/ARB, spironolactone for aldosterone antagonists, and frusemide for loop diuretics) and titration protocols were based on National Institute of Clinical Excellence guidelines for HF (guideline 5).

Results Between October 2009 and Jun 2010, 74 patients (age: $67 \pm 11 \mathrm{yrs}, 86 \%$ male) underwent implantation of a CRT device. All

Abstract 75 Table 1 Heart Failure nurse supervised use of medications before and 6 months After CRT

\begin{tabular}{|c|c|c|c|}
\hline & Pre-CRT & Post-CRT & p Value \\
\hline \multicolumn{4}{|c|}{$\beta$-Blocker: exemplar bisoprolol } \\
\hline Frequency of use & $78 \%$ & $88 \%$ & \multirow[t]{2}{*}{$<0.01$} \\
\hline Daily dose equivalent & $5.3 \pm 3.1 \mathrm{mg} /$ day & $6.9 \pm 3.2 \mathrm{mg} / \mathrm{day}$ & \\
\hline \multicolumn{4}{|c|}{ ACE-I/ARB: exemplar lisinopril } \\
\hline Frequency of use & $93 \%$ & $93 \%$ & \multirow[t]{2}{*}{0.02} \\
\hline Daily dose equivalent & $11.8 \pm 4.5 \mathrm{mg} / \mathrm{day}$ & $12.4 \pm 3.8 \mathrm{mg} / \mathrm{day}$ & \\
\hline \multicolumn{4}{|c|}{ Aldosterone Antagonist: exemplar spironolactone } \\
\hline Frequency of use & $32 \%$ & $28 \%$ & \multirow[t]{2}{*}{0.32} \\
\hline Daily dose equivalent & $16.6 \pm 6.2 \mathrm{mg} /$ day & $15.5 \pm 7.3 \mathrm{mg} /$ day & \\
\hline \multicolumn{4}{|c|}{ Cardiac Glycoside: exemplar digoxin } \\
\hline Frequency of use & $43 \%$ & $48 \%$ & \multirow[t]{2}{*}{$<0.01$} \\
\hline Daily dose equivalent & $83.8 \pm 11.4 \mu \mathrm{g} / \mathrm{day}$ & $96.1 \pm 13.6 \mu \mathrm{g} /$ day & \\
\hline \multicolumn{4}{|c|}{ Loop Diuretic: exemplar frusemide } \\
\hline Frequency of use & $100 \%$ & $100 \%$ & \multirow[t]{2}{*}{$<0.01$} \\
\hline Daily dose equivalent & $63.0 \pm 5.8 \mathrm{mg} / \mathrm{day}$ & $48.1 \pm 8.4 \mathrm{mg} / \mathrm{day}$ & \\
\hline
\end{tabular}

patients attended the pre and post CRT nurse clinic to optimise medical therapies and provide adjunctive HF support. Abstract 75 table 1 describes the frequency of use and daily dose equivalent of each class of medication used in the patients prior to and 6 months after device implantation. The frequency of $\beta \mathrm{B}$ and digoxin use increased by $10 \%$ and $5 \%$ respectively. In addition, the dose of $\beta B$, ACE-I/ARB, and digoxin significantly increased, while the dose of loop diuretics significantly reduced in the 6 months after CRT implantation.

Conclusions The beneficial haemodynamic and pacing profiles provided by CRT offer important opportunities to further optimise heart failure medications after device implantation. In a dedicated nurse-led CRT follow-up clinic, we successfully initiated $\beta$ blockers and digoxin in previously naive patients, and significantly uptitrated the doses of $\beta$ blockers, ACE-I/ARB, and digoxin, while significantly reducing loop diuretic use in the 6 months after device implantation.

\section{EXPANDING THE ROLE OF CARDIAC CARE UNIT NURSES TO REDUCE TIME TO TREATMENT FOR PATIENTS REOUIRING PRIMARY ANGIOPLASTY}

doi:10.1136/heartjnl-2011-300198.76

S Young, G Pretsell, A Gibbins, G Dixon, A de Belder. Royal Sussex County Hospital, Brighton, UK

Introduction In Brighton, UK, 24-h Primary Angioplasty has been used for the treatment of ST segment elevation myocardial infarction (STEMI) since October 2008, with local patients being admitted via the Accident and Emergency (A\&E) department. With the publication of the National Infarct Angioplasty project report (DH 2008) it was evident that direct admission into the cardiac catheter lab from the ambulance could further reduce time to treatment. Call to Balloon time (CTBT) $<150$ mins is a nationally recognised indicator measuring the time the patient first calls for professional help (usually the ambulance) to the opening of the coronary artery on the catheter lab Abstract 76 table 1.

\section{Abstract 76 Table 1}

\begin{tabular}{lll}
\hline & $\%$ CTBT $\leq 150$ mins & Median CTBT \\
\hline Financial Year 2009-2010 & 59/78 76\%* & 125 mins \\
Quarter 1 (April-June 2010) & $32 / 3689 \%$ & 111 mins \\
Quarter 2 (July-September 2010) & $44 / 4598 \% *$ & 99 mins \\
\hline
\end{tabular}

${ }^{*} p=0.0013$.

Methods The on-call cardiology team are non-resident out of hours It was therefore agreed the point of contact and immediate decision making would lie with the Cardiac Care Unit (CCU) nurses. A pathway was developed following consultation with the multidisciplinary team at an educational and mapping day, and risks were addressed. It was agreed that the ambulance crew would telephone the CCU nurse who would review the clinical history and the telemetry ECG. They would then make the decision to activate the catheter lab team. Patient Group Directions for the administration of GTN, diamorphine, metoclopramide and clopidogrel were developed so that immediate treatment could be delivered by the CCU nurse without medical prescription before the cardiac catheter lab team arrived, if required. The nurses were trained in their use and assessed as competent. Nurses were already competent in ECG interpretation, defibrillation, cannulation and venepuncture. Nursing documentation was developed to prioritise the patient's emergency care. CTBT were monitored. 
Results Following the implementation of the direct entry pathway in May 2010 the CTBT for all patients admitted direct to our hospital have reduced. This is statistically significant when looking at Quarter 2 results from baseline. Patient safety has not been compromised. Patients who were admitted directly have been asked about their experience and if anything could be done differently from their perspective. They have said:

- The process is quick which is good from their perspective

- They are fully informed

- The ambulance crews deal with them competently

- The lab staff are waiting for their arrival.

Conclusions The CCU nurses have embraced this development and expansion of their nursing practice, allowing major changes to be made to the Primary Angioplasty pathway within the existing infrastructure, despite the challenges of working within the complex nature of traditional geographical referral patterns. Along with the work of all members of the multi disciplinary team this has significantly reduced times to treatment for patients.

\section{SCREENING FIRST DEGREE RELATIVES FOR HYPERTROPHIC CARDIOMYOPATHY: 12-MONTH EXPERIENCE OF A CARDIO- GENETICS NURSE SERVICE}

doi:10.1136/heartjnl-2011-300198.77

\author{
${ }^{1} \mathrm{~S}$ Finch, ${ }^{2} \mathrm{~S}$ Russell, ${ }^{1} \mathrm{D}$ Kumar, ${ }^{1} \mathrm{Z}$ R Yousef. ${ }^{1}$ University Hospital of Wales, Cardiff, UK; \\ ${ }^{2}$ Wales Heart Research Institute, Cardiff, UK
}

Introduction Hypertrophic cardiomyopathy (HCM) is an autosomally transmitted cardiomyopathy with an estimated gene prevalence of 1:500, and an important cause of sudden cardiac death. Screening to identify at risk first degree relatives is therefore recommended. The British Heart Foundation (BHF) recently funded nine Nationwide cardio-genetic nurses to support local initiatives. Our application for a nurse was successful and we present our 12-month experience of HCM screening.

Methods We mapped the course of patients with suspected HCM referred to our tertiary heart muscle clinic which serves a population of 1.4 million. Following phenotype confirmation, a family tree and contact details from the index case were recorded by the cardiogenetic nurse. The index case was given literature to pass onto at risk relatives. The information pack included an open invitation (referral via primary care) to attend for screening. For relatives residing outside our catchment area screening was arranged via links with the BHF cardio-genetic network and other health care providers. Relatives domiciled outside UK were given our details with offers to support screening. Throughout, strict adherence to patient confidentiality was maintained.

Results Over 12 months, 64 index HCM cases presented to our heart muscle clinic. Pedigree analysis identified 221 first degree relatives at risk of carrying the HCM gene; mean index-to-at RR: 1-to-3.4 (range 0-14 subjects). Of the 221 at risk subjects, 71 (19 through paediatrics) have undergone screening through clinical assessment at our unit with plans for long-term 2-5 yearly follow-up in view of variable gene penetrance. Of the 71 screened subjects, 15 were newly diagnosed with HCM. Newly diagnosed HCM patients underwent further risk stratification for sudden cardiac death; where we identified 3 patients at high risk $(\geq 2$ conventional high sudden death risk factors). After appropriate counselling, these 3 patients have received primary prevention defibrillators. Despite our approach, 52 subjects remain unscreened (Abstract 77 table 1), either due to complex family relationships $(n=14)$, personal preference $(n=28)$ and/or geographical/logistical reasons $(n=10)$.
Abstract 77 Table 1 Screening outcomes of 221 at risk subjects identified from 64 index cases of hypertrophic cardiomyopathy

\begin{tabular}{lc}
\hline & Number of Patients \\
\hline New screening initiated (local heart muscle clinic) & 52 \\
New screening initiated (local paediatric clinic) & 19 \\
New screening initiated (out of area service) & 6 \\
Pre-existing screening in place & 63 \\
Personal preference (declined screening) & 28 \\
Awaiting response from subject (literature delivered) & 19 \\
Complex family relationships (unable to deliver literature) & 14 \\
Geographical/Logistical constraints & 10 \\
Subject deceased (non-hypertrophic cardiomyopathy) & 3 \\
Subject deceased (hypertrophic cardiomyopathy) & 7 \\
\hline
\end{tabular}

Conclusions Proactive screening for HCM can be effectively facilitated by cardio-genetic nurse services. Each new index case generates 3-4 at risk relatives who require long-term surveillance. Of 71 asymptomatic at risk subjects screened in our unit, we diagnosed 15 new cases of HCM, and 3 patients at high risk of sudden cardiac death who subsequently received primary prevention defibrillator implantation.

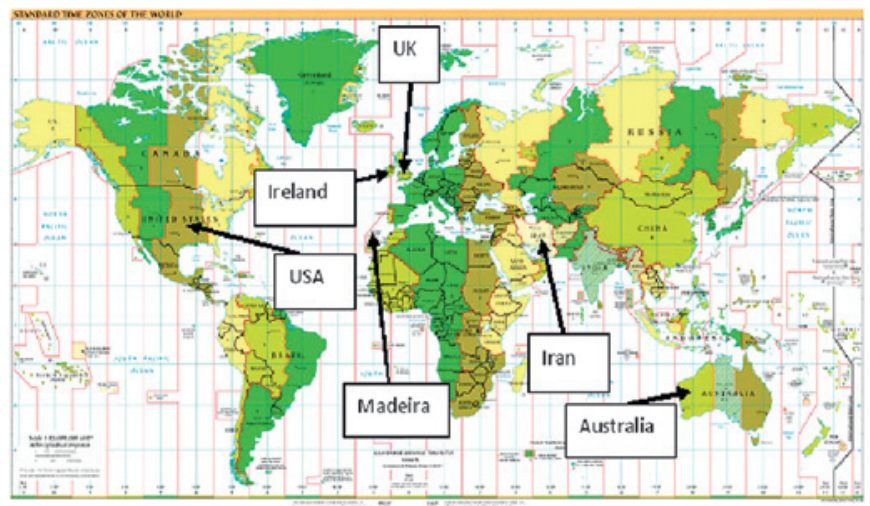

Abstract 77 Figure 1

\section{FIRST YEAR EXPERIENCE OF A DEDICATED "RADIAL LOUNGE" FOR PATIENTS UNDERGOING ELECTIVE PERCUTANEOUS CORONARY PROCEDURES}

doi:10.1136/heartjnl-2011-300198.78

S Brewster, R Weerackody, K Khimdas, A Little, N Cleary, A Penswick, M Rothman, A Archbold. London Chest Hospital, London, UK

Introduction The potential to achieve safe early mobilisation and same day discharge on a consistent basis after radial artery access has provided us with the opportunity to make a step change in the way we deliver elective care to patients undergoing percutaneous coronary procedures. We designed a dedicated "radial lounge" to accommodate patients before and after their procedure with the aim of minimising the feeling of "hospitalisation" that accompanies most encounters with health services. The lounge is a day case unit that has no beds, only chairs, and televisions but no cardiac monitors. Patients remain in their clothes throughout their hospital visit. Here we report our first year's experience of this facility. Methods: The study population comprised all patients who attended the radial lounge between July 2009-June 2010 for coronary angiography or percutaneous coronary intervention (PCI). Patients were suitable for the radial lounge if they were elective cases who had a satisfactory radial pulse and no pre-procedure contraindication to 